\title{
Retraction: Blockage of transdifferentiation from fibroblast to myofibroblast in experimental ovarian cancer models Qin Yao ${ }^{1,2,3}$, Xun Qu ${ }^{4}$, Qifeng Yang4 ${ }^{4}$ David A Good ${ }^{1}$, Shuzhen Dai ${ }^{3}$, Beihua Kong ${ }^{2}$ and Ming Q Wei*1
}

Address: ${ }^{1}$ Division of Molecular and Gene Therapies, Griffith Institute for Health and Medical Research, School of Medical Science, Griffith University, Gold Coast campus, Southport, Qld 4222, Australia, ${ }^{2}$ Department of Obstetrics and Gynaecology, Qilu Hospital, Shandong University, Ji'nan 250012, Shandong, PR China, 3Department of Obstetrics and Gynaecology, Affiliated Hospital of Qingdao University, Qing'dao 266003, Shandong, PR China and "Institute of Basic Medical Sciences, Qilu Hospital, Shandong University, Ji'nan 250012, Shandong, PR China

Email: Qin Yao - Dr.yaoqin@yahoo.com.cn; Xun Qu - quxun@yahoo.com.cn; Qifeng Yang - yangqifeng@yahoo.com.cn; David A Good - d.good@griffith.edu.au; Shuzhen Dai - Shuzhendai@yahoo.com.cn; Beihua Kong - kongbeihua@hotmail.com; Ming Q Wei* - m.wei@griffith.edu.au

* Corresponding author

Molecular Cancer 2009, 8:84 doi:10.1 186/1476-4598-8-84

This article is available from: http://www.molecular-cancer.com/content/8/I/84

(C) 2009 Yao et al; licensee BioMed Central Ltd.

This is an Open Access article distributed under the terms of the Creative Commons Attribution License (http://creativecommons.org/licenses/by/2.0), which permits unrestricted use, distribution, and reproduction in any medium, provided the original work is properly cited.

\section{Retraction}

This article [1] was submitted to Molecular Cancer following the acceptance of an article in Oncology Reports [2], published in September 2009. The two articles were produced from the same data and as a result of miscommunication between authors contained extensive overlap. As such the authors would like to retract the Molecular Cancer article. The authors would like to apologise for any inconvenience this may have caused to the editorial staff and readers.

\section{References}

I. Yao Q, Qu X, Yang Q, Good DA, Dai S, Kong B, Wei MQ: Blockage of transdifferentiation from fibroblast to myofibroblast in experimental ovarian cancer models. Mol Cancer 2009, 8:78.

2. Yao Q, Qu X, Yang Q, Wei M, Kong B: CLIC4 mediates TGF- $\beta$ Iinduced fibroblast-to-myofibroblast transdifferentiation in ovarian cancer. Oncol Rep 2009, 22:54I-548. 